\title{
Hamiltonian formalism for space-time noncommutative theories
}

\author{
Joaquim Gomis, ${ }^{1,2}$ Kiyoshi Kamimura, ${ }^{3}$ and Josep Llosa ${ }^{4}$ \\ ${ }^{1}$ Theory Division, $\mathrm{CH}-1211$ Geneva 23, Switzerland \\ ${ }^{2}$ Departament ECM, Facultat de Física, Universitat de Barcelona and Institut de Física d'Altes Energies, Diagonal 647, E-08028 \\ Barcelona, Spain \\ ${ }^{3}$ Department of Physics, Toho University, Funabashi 274-8510, Japan \\ ${ }^{4}$ Departament de Física Fonamental, Universitat de Barcelona, Diagonal 647, E-08028 Barcelona, Spain
}

(Received 5 July 2000; published 12 January 2001)

\begin{abstract}
Space-time non-commutative theories are nonlocal in time. We develop the Hamiltonian formalism for non-local field theories in $d$ space-time dimensions by considering auxiliary $(d+1)$-dimensional field theories which are local with respect to the evolution time. The Hamiltonian path integral quantization is considered and the Feynman rules in the Lagrangian formalism are derived. The case of non-commutative $\phi^{3}$ theory is considered as an example.
\end{abstract}

DOI: 10.1103/PhysRevD.63.045003

PACS number(s): 11.10.Ef, 11.10.Lm, 11.15.Kc

\section{INTRODUCTION}

Space-time non-commutative field theories have peculiar properties due to their acausal behavior $[1,2]$ and lack of unitarity [3]. In Ref. [4] it has been shown that there is a relation between the lack of unitarity and the obstruction to finding a decoupling limit of string theory in an electromagnetic background [5-10]. These theories have an infinite number of temporal and spatial derivatives, and therefore are non-local in time and space $[11,12]$. The initial value problem of a non-local theory requires one to give a trajectory or a finite piece of it [13]. The Euler-Lagrange (EL) equation is a constraint in the space of trajectories.

The Hamiltonian formalism for non-local theories was presented in [15]. In this paper we improve the formalism by clarifying the relation among the Lagrangian and Hamiltonian structures. We first consider an equivalent theory in a space-time of one dimension higher than that of the original theory. This space has "two times" and the dynamics is described in such a way that the evolution is local with respect to one of the times. For this equivalent theory one can construct the Hamiltonian. A characteristic feature of the Hamiltonian formalism for non-local theories is that it contains the EL equations as Hamiltonian constraints.

The Hamiltonian path integral for the $(d+1)$-dimensional field theory is constructed. The Lagrangian path integral formalism for the $d$ dimensional theory is obtained by integrating out the momenta.

We apply the Hamiltonian formalism to time-like and light-like non-commutative theories [4]. As an example we consider the case of non-commutative $\phi^{3}$ theory in $d$ dimensions with space-time non-commutativity. The action contains the free Klein-Gordon Lagrangian and the interaction Lagrangian $L_{i}=(-g / 3 !) \int d \vec{x} \phi^{*} \phi^{*} \phi$, where * refers to the Moyal product. We construct the Hamiltonian in $d+1$ dimensions. In the path integral quantization we get the Feynman rules that coincide with those used in Refs. [1-3]. The theory is unitary at the classical level (tree level) but is not unitary at one loop [3]. This analysis should shed new light on the structure of these theories. Knowledge of the Hamiltonian of time-like and light-like non-commutative field theories could also be useful to study the energy of their solitons.

\section{EULER-LAGRANGE EQUATIONS FOR NON-LOCAL THEORIES}

Unlike standard Lagrangians, which depend on the values of a finite number of derivatives at a given time, $q(t)$, $\dot{q}(t), \ldots, q^{(n)}(t)$, a non-local Lagrangian depends on a whole piece of the trajectory $q(t+\lambda)$, for all values of $\lambda$, that is, $L^{\mathrm{non}}(t)=L([q(t+\lambda)])$. At best it can be written as a function of all time derivatives $q^{(j)}(t), j=0,1,2, \ldots$, at the same $t$. This means that the analogue of the tangent bundle for Lagrangians depending on positions and velocities is infinite dimensional. The action is

$$
S[q]=\int d t L^{\mathrm{non}}(t)
$$

The EL equation is obtained as the variation of functional (1) and is given by

$$
\int d t E\left(t, t^{\prime} ;[q]\right)=0
$$

where $E\left(t, t^{\prime} ;[q]\right)=\delta L^{\mathrm{non}}(t) / \delta q\left(t^{\prime}\right)$.

The EL equation must be understood as a functional relation to be satisfied by physical trajectories. It is not a differential system, as one is used to finding for local Lagrangians. In the latter case, the theorems of the existence and uniqueness of solutions enable one to interpret the EL equation as ruling the time evolution of the system, whose state at every instant of time is represented by a point in the space of initial data, e.g., $J_{L}=\left\{q, \dot{q}, \ldots, q^{(2 n-1)}\right\}$, for a local Lagrangian of order $n$.

In the non-local case, if we denote the space of all possible trajectories as $J=\{q(\lambda), \lambda \in R\}$, Eq. (2) is a Lagrangian constraint defining the subspace $J_{R} \subset J$ of physical trajectories. 


\section{III. (1+1)-DIMENSIONAL FIELD THEORY DESCRIPTION OF NON-LOCAL THEORIES}

Nevertheless, if we insist in defining a "time evolution" $T_{t}$ for a given initial trajectory $q(\lambda)$, a natural choice is

$$
q(\lambda) \stackrel{T_{t}}{\rightarrow} q(\lambda+t)
$$

We shall hence introduce new dynamical variables $Q(t, \lambda)$ such that

$$
Q(t, \lambda)=q(\lambda+t)
$$

Thus, $t$ is the "evolution" parameter and $\lambda$ is a continuous parameter indexing the degrees of freedom. These new variables follow the evolution (4) above, and $Q(0, \lambda)$ can be seen as initial data in the local $(1+1)$-dimensional field theory.

In differential form, condition (4) reads

$$
\dot{Q}(t, \lambda)=Q^{\prime}(t, \lambda),
$$

where the overdot and prime respectively stand for $\partial_{t}$ and $\partial_{\lambda}$.

We then consider the Hamiltonian system for the $(1+1)$ dimensional field $Q$ with the Hamiltonian

$$
H(t,[Q, P])=\int d \lambda P(t, \lambda) Q^{\prime}(t, \lambda)-\widetilde{L}(t,[Q]),
$$

where $P$ is the canonical momentum of $Q$. The phase space is thus $T^{*} J$ with the fundamental Poisson brackets

$$
\left\{Q(t, \lambda), P\left(t, \lambda^{\prime}\right)\right\}=\delta\left(\lambda-\lambda^{\prime}\right) .
$$

In the Hamiltonian (6), $\widetilde{L}(t,[Q])$ is a functional defined by

$$
\widetilde{L}(t,[Q]):=\int d \lambda \delta(\lambda) \mathcal{L}(t, \lambda) .
$$

The "density" $\mathcal{L}(t, \lambda)$ is constructed from $L^{\text {non }}(t)$ by replacing $q(t)$ by $Q(t, \lambda)$, the $t$ derivatives of $q(t)$ by $\lambda$ derivatives of $Q(t, \lambda)$ and $q(t+\rho)$ by $Q(t, \lambda+\rho)$. In this construction of the Hamiltonian $\lambda$ inherits the signature of the original time $t$ and is a time-like coordinate. Furthermore, the symmetry of the original Lagrangian is realized canonically in the enlarged space [14]. Note that $\mathcal{L}(t, \lambda)$ is local in $t$ and is nonlocal in $\lambda$. $H$ depends linearly on $P(t, \lambda)$ but does not depend on $\dot{Q}(t, \lambda)$.

The relation (5) naturally arises as the first Hamilton equation for Eq. (6). However, there is no a priori relationship between $P(t, \lambda)$ and $Q(t, \lambda)$, unlike what happens in the local case, and the second Hamilton equation

$$
\dot{P}(t, \lambda)=P^{\prime}(t, \lambda)+\frac{\delta \tilde{L}(t,[Q])}{\delta Q(t, \lambda)}
$$

does not imply any further restriction on $Q(t, \lambda)$. Thus, the Hamiltonian system (6) on $T^{*} J$ is not so far equivalent to the non-local Lagrangian system of $L^{\text {non }}(t)$.
Now, instead of taking the whole phase space $T^{*} J$, we shall restrict ourselves to the subspace defined by the 1-parameter set of primary constraints [15]:

$$
\varphi(t, \lambda,[Q, P]) \equiv P(t, \lambda)-F(t, \lambda,[Q]) \approx 0
$$

with

$$
F(t, \lambda,[Q]):=\int d \sigma \chi(\lambda,-\sigma) \mathcal{E}(t ; \sigma, \lambda),
$$

where $\mathcal{E}(t ; \sigma, \lambda)$ and $\chi(\lambda,-\sigma)$ are defined by

$$
\mathcal{E}(t ; \sigma, \lambda)=\frac{\delta \mathcal{L}(t, \sigma)}{\delta Q(t, \lambda)}, \quad \chi(\lambda,-\sigma)=\frac{\epsilon(\lambda)-\epsilon(\sigma)}{2}
$$

Here $\epsilon(\lambda)$ is the sign distribution. The symbols " $\equiv$ ", and " $\approx$ " respectively stand for "strong", and "weak" equality.

Further constraints are generated by requiring the stability of the primary ones. In the first step, we obtain

$$
\dot{\varphi}(t, \lambda,[Q, P]) \equiv \varphi^{\prime}(t, \lambda,[Q, P])+\delta(\lambda) \psi_{0}(t,[Q]) \approx 0
$$

where

$$
\psi_{0}(t,[Q]):=\int d \sigma \mathcal{E}(t ; \sigma, 0) \approx 0
$$

is the secondary constraint. Further constraints then follow by successive time differentiations of $\psi_{0}$. They can be written all together in a condensed form as

$$
\psi(t, \lambda,[Q]) \equiv \int d \sigma \mathcal{E}(t ; \sigma, \lambda) \approx 0
$$

Therefore, the constrained Hamiltonian system defined by the Hamiltonian (6) and the primary constraints (10) resides in a reduced phase space $\Gamma \subset T^{*} J$ defined by Eqs. (10) and (14). Taking into account Eq. (4), the constraint (14) reduces to the EL equation (2) obtained from $L^{\text {non }}(t)$.

The constraints (10) and (14) belong to the second class in non-singular systems. In the next section we will show explicitly, for (non-singular) higher derivative Lagrangian system of order $n$, that they are used to reduce the phase space to $2 n$ dimensions, reproducing the canonical Ostrogradski formalism [16]. Our formalism developed here turns out to be a generalization of the Ostrogradski formalism to the case of infinite order derivative theories. The infinite chain of second class constraints has also appeared in the description of boundary conditions as constraints [17]. Summarizing, the equivalence has been built in the $(1+1)$-dimensional Hamiltonian formalism of local field theories through the constraints (10) and (14). This type of equivalence between the Hamiltonian and Lagrangian formalism is different from the one in local theories [18]. 


\section{NON-SINGULAR HIGHER ORDER DERIVATIVE THEORIES}

Here we would like to derive both the Lagrangian and Hamiltonian formalisms for non-singular higher order derivative theories from the Hamiltonian formalism of nonlocal theories developed in the last section [19].

Let us consider a regular higher derivative theory described by the Lagrangian $L\left(q, \dot{q}, \ddot{q}, \ldots, q^{(n)}\right)$ and write the expressions obtained in the previous section for the non-local Lagrangian. As we embed the higher order theory in the non-local setting we start with the infinite dimensional phase space $T^{*} J(t)=\{Q(t, \lambda), P(t, \lambda)\}$. They are assumed to be expanded in the Taylor basis [20] as

$$
\begin{aligned}
& Q(t, \lambda) \equiv \sum_{m=0}^{\infty} e_{m}(\lambda) q^{m}(t), \\
& P(t, \lambda) \equiv \sum_{m=0}^{\infty} e^{m}(\lambda) p_{m}(t),
\end{aligned}
$$

where $e^{l}(\lambda)$ and $e_{l}(\lambda)$ are orthonormal bases:

$$
e^{l}(\lambda)=\left(-\partial_{\lambda}\right)^{l} \delta(\lambda), \quad e_{l}(\lambda)=\frac{\lambda^{l}}{l !} .
$$

The coefficients in Eqs. (15) are new canonical variables,

$$
\left\{q^{m}(t), p_{n}(t)\right\}=\delta^{m}{ }_{n},
$$

and the Hamiltonian (6) is

$$
H(t)=\sum_{m=0}^{\infty} p_{m}(t) q^{m+1}(t)-L\left(q^{0}, q^{1}, \ldots, q^{n}\right) .
$$

The momentum constraint (10) becomes an infinite set of constraints:

$$
\varphi_{m}(t)=p_{m}(t)-\sum_{l=0}^{n-m-1}\left(-D_{t}\right)^{l} \frac{\partial L(t)}{\partial q^{l+m+1}(t)} \approx 0,
$$

where

$$
D_{t}=\sum_{r=0} q^{r+1} \frac{\partial}{\partial q^{r}}
$$

On the other hand, the constraint $\psi$ in Eq. (14) in terms of the Taylor basis becomes

$$
\psi^{m}(t) \equiv\left(D_{t}\right)^{m}\left[\sum_{l=0}^{n}\left(-D_{t}\right)^{l} \frac{\partial L(t)}{\partial q^{l}(t)}\right] \approx 0 .
$$

These constraints (19) and (21) are second class and are used to reduce the infinite dimensional phase space to a finite one, leading to the ordinary Ostrogradski Hamiltonian formalism. The operator $D_{t}$ defined in Eq. (20) becomes a time evolution operator for $q$ 's using the first set of the Hamilton equation

$$
\dot{q}^{r}=q^{r+1} .
$$

Using this in Eq. (19) the constraints $\varphi_{m}(0 \leqslant m \leqslant n-1)$ coincide with the definition of the Ostragradsky momenta:

$$
p_{m} \sim \sum_{l=0}^{n-m-1}\left(-\partial_{t}\right)^{l} \frac{\partial L(t)}{\partial\left(\partial_{t}^{l+m+1} q(t)\right)} \quad(0 \leqslant m \leqslant n-1) .
$$

Now they can be solved for $q^{l}(n \leqslant l \leqslant 2 n-1)$ as functions of the canonical pairs $\left\{q^{j}, p_{j}\right\} \quad(0 \leqslant j \leqslant n-1)$

$$
\begin{gathered}
q^{l} \approx q^{l}\left(q^{0}, q^{1}, \ldots, q^{n-1}, p_{0}, p_{1}, \ldots, p_{n-1}\right) \\
(n \leqslant l \leqslant 2 n-1) .
\end{gathered}
$$

They are combined with the constraints $\varphi_{l}(n \leqslant l \leqslant 2 n$ $-1)$,

$$
\varphi_{l}=p_{l} \approx 0 \quad(n \leqslant l \leqslant 2 n-1)
$$

to form a second class set and can be used to eliminate the canonical pairs $\left\{q^{l}, p_{l}\right\}(n \leqslant l \leqslant 2 n-1)$.

If we take into account Eq. (22) the constraint (21) for $m=0$ is the Euler-Lagrange equation for the original higher derivative Lagrangian:

$$
\psi^{0} \sim \sum_{l=0}^{n}\left(-\partial_{t}\right)^{l} \frac{\partial L(t)}{\partial\left(\partial_{t}^{l} q(t)\right)}=0 .
$$

The constraints (21) for $m>0$ are the time derivatives of the Euler-Lagrange equation (26) expressed in terms of $q$ 's. For a non-singular theory, all the constraints (21) can be rewritten as

$$
q^{l}-q^{l}\left(q^{0}, q^{1}, \ldots, q^{n-1}, p_{0}, p_{1}, \ldots, p_{n-1}\right) \approx 0 \quad(l \geqslant 2 n)
$$

and can be paired with the constraints $\varphi_{l}(l \geqslant 2 n)$,

$$
\varphi_{l}=p_{l} \approx 0 \quad(l \geqslant 2 n),
$$

forming second class constraints. They are used to eliminate the canonical pairs $\left\{q^{l}, p_{l}\right\} \quad(l \geqslant 2 n)$.

In this way the infinite dimensional phase space is reduced to a finite dimensional one. The reduced phase space is coordinated by $T^{*} J^{n}=\left\{q^{l}, p_{l}\right\}$ with $l=0,1, \ldots, n-1$. All the constraints are second class and we use the iterative property of Dirac brackets. The Dirac brackets for these variables have the standard form

$$
\left\{q^{m}, p_{n}\right\}^{*}=\delta^{m}{ }_{n}, \quad\left\{q^{m}, q^{n}\right\}^{*}=\left\{p_{m}, p_{n}\right\}^{*}=0 .
$$

The Hamiltonian (6) in the reduced space is given by

$$
H(t)=\sum_{m=0}^{n-1} p_{m}(t) q^{m+1}(t)-L\left(q^{0}, q^{1}, \ldots, q^{n}\right)
$$

where $q^{n}$ is expressed using Eq. (24) as a function of the reduced variables in $T^{*} J^{n}$. Note that if we consider the limit $n$ going to infinity, the constraints (19) and (21) do not allow 
one, in general, to reduce the dimensionality of the infinite dimensional phase space of the non-local system via Dirac brackets.

\section{SYMPLECTIC FORMULATION OF THE EULER-LAGRANGE EQUATION}

The Hamiltonian formalism presented in the last sections can be cast into a symplectic form as follows. The Poisson brackets (7) correspond to the symplectic two-form $\Omega$ $\in \Lambda^{2}\left(T^{*} J\right)$ :

$$
\Omega=\int d \lambda \delta P(t, \lambda) \wedge \delta Q(t, \lambda),
$$

where $\delta$ stands for the functional exterior derivative.

In the constrained phase space $\Gamma_{1} \subset T^{*} J$ defined by Eq. (10) only, the induced (pre)symplectic form is

$$
\Omega_{1}=\frac{1}{2} \int d \lambda d \lambda^{\prime} \omega\left(t ; \lambda, \lambda^{\prime},[Q]\right) \delta Q(t, \lambda) \wedge \delta Q\left(t, \lambda^{\prime}\right)
$$

where

$$
\omega\left(t ; \lambda, \lambda^{\prime},[Q]\right)=\chi\left(\lambda^{\prime},-\lambda\right) \int d \sigma \frac{\delta \mathcal{E}(t ; \sigma, \lambda)}{\delta Q\left(t, \lambda^{\prime}\right)} .
$$

The induced Hamiltonian is

$$
H_{1}(t,[Q])=\int d \lambda F(t, \lambda,[Q]) Q^{\prime}(t, \lambda)-\widetilde{L}(t,[Q])
$$

The generator of the time evolution (4) is the vector field

$$
X([Q])=\int d \lambda \dot{Q}(t, \lambda) \frac{\delta}{\delta Q(t, \lambda)}
$$

Now, $i(X) \Omega_{1}+\delta H_{1}=0$ gives a first order formulation of the EL equation. Indeed a short calculation yields

$$
\begin{aligned}
i(X) \Omega_{1}+\delta H_{1}= & -\int d \sigma \mathcal{E}(t ; \sigma, 0) \delta Q(t, 0) \\
& +\int d \lambda d \lambda^{\prime} \delta Q\left(t, \lambda^{\prime}\right) \\
& \times\left[\dot{Q}(t, \lambda)-Q^{\prime}(t, \lambda)\right] \omega\left(t ; \lambda, \lambda^{\prime}\right),
\end{aligned}
$$

whence the evolution (5) and the EL equation (2) follow from it.

\section{PATH INTEGRAL QUANTIZATION}

Let us consider the Hamiltonian path integral quantization of the $(1+1)$-dimensional field theory associated with the Hamiltonian (6) for $L^{\text {non }}(t)$. The path integral is given by

$$
\begin{aligned}
& \int \quad[d P(t, \lambda)][d Q(t, \lambda)] \mu \\
& \quad \times \exp \left(i \int d t d \lambda \left\{P(t, \lambda)\left[\dot{Q}(t, \lambda)-Q^{\prime}(t, \lambda)\right]\right.\right. \\
& \quad+\widetilde{L}(t) \delta(\lambda)\}) .
\end{aligned}
$$

The integration is performed over the reduced phase space $\Gamma$ and the measure $[21,22] \mu$ is

$$
\mu=\operatorname{det}\left(\begin{array}{ll}
\{\varphi, \varphi\} & \{\varphi, \psi\} \\
\{\psi, \varphi\} & \{\psi, \psi\}
\end{array}\right) \delta(\varphi) \delta(\psi)
$$

First we consider the non-singular higher derivative Lagrangian system of order $n$. From the discussions of Sec. IV the constraints are arranged in a set in which the canonical variables $\left(q^{j}, p_{j}\right)$ for $j \geqslant n$ are expressed in terms of the ones for $0 \leqslant j \leqslant n-1$. The measure becomes

$$
\mu=\prod_{j=n}^{2 n-1}\left\{\delta\left(p_{j}\right) \delta\left(q^{j}-\cdots\right)\right\} \prod_{k=2 n}^{\infty}\left\{\delta\left(p_{k}\right) \delta\left(q^{k}-\cdots\right)\right\}
$$

where $\cdots$ terms are given as functions of $\left(q^{i}, p_{i}\right)(i$ $=0, \ldots, n-1)$. Integrating over $\left(q^{i}, p_{i}\right)(i \geqslant n)$ Eq. (37) becomes

$$
\begin{aligned}
& \int \prod_{i=0}^{n-1} d q^{i} d p_{i} \exp \left(i \int d t \sum_{i=0}^{n-1} p_{i}\left(\dot{q}^{i}-q^{i+1}\right)\right. \\
& \left.\quad+L\left(q^{0}, \ldots, q^{n}\right)\right)
\end{aligned}
$$

where $q^{n}$ is given as a function of $\left(q^{i}, p_{i}\right)(i=0, \ldots, n-1)$. This is the Hamiltonian path integral of the Ostrogradski formalism. If we assume that non-local systems can be regarded as the infinite limit of $n$, the higher derivative system (40) becomes, by taking $n \rightarrow \infty$,

$$
\begin{aligned}
& \int \quad[d P(t, \lambda)][d Q(t, \lambda)] \\
& \quad \times \exp \left(i \int d t d \lambda \left\{P(t, \lambda)\left[\dot{Q}(t, \lambda)-Q^{\prime}(t, \lambda)\right]\right.\right. \\
& \quad+\widetilde{L}(t) \delta(\lambda)\}),
\end{aligned}
$$

where $Q$ and $P$, which are $n \rightarrow \infty$ of $\left(q^{i}, p_{i}\right)(i=0, \ldots, n$ $-1)$, are not restricted by the constraints in contrast to Eq. (37).

If we integrate out the momenta and use $\delta(\dot{Q}(t, \lambda)$ $\left.-Q^{\prime}(t, \lambda)\right)$, we get

$$
\int[d q(t)] \exp \left(i \int d t L^{\mathrm{non}}(t)\right),
$$


which is the Lagrangian path integral formulation for the non-local theory.

\section{APPLICATION TO SPACE-TIME NON-COMMUTATIVE $\phi^{3}$ THEORY}

Space-time non-commutative theories have peculiar properties due to their acausal behavior and lack of unitarity. Here we would like to use the previous formalism to study the question of unitarity in these theories.

To fix the ideas we consider a non-commutative $\phi^{3}$ theory with arbitrary non-commutativity in $d$ dimensions. The Lagrangian density is given by

$$
\begin{aligned}
\mathcal{L}^{\text {non }}\left(x^{\mu}\right)= & \frac{1}{2} \partial_{\mu} \phi(x) \partial^{\mu} \phi(x)-\frac{m^{2}}{2} \phi(x)^{2} \\
& -\frac{g}{3 !} \phi(x) * \phi(x) * \phi(x)
\end{aligned}
$$

where $*$ is the star product defined by using a general antisymmetric background $\theta^{\mu \nu}$ :

$$
f(x) * g(x)=\left[e^{i\left(\theta^{\mu \nu} / 2\right) \partial_{\mu}^{\alpha} \partial^{\beta}} f(x+\alpha) g(x+\beta)\right]_{\alpha=\beta=0} .
$$

The EL equation is

$$
\left(\square-m^{2}\right) \phi(x)-\frac{g}{2 !} \phi(x) * \phi(x)=0
$$

$x^{0}$ in Eqs. (43)-(45) will be denoted as $t$ hereafter. We introduce a "new coordinate $x^{0}$, which plays the role of $\lambda$ in the previous discussion and introduce the field $Q\left(t, x^{\mu}\right)$ in $d+1$ dimensions. Now $t$ is regard as "evolution time" and $x^{\mu}:=\left(x^{0}, \vec{x}\right)$ is a continuous Lorentzian index. Our metric conventions are $\eta_{t t}=\eta_{00}=-1, \eta_{i i}=+1$. The relation (4) in this case is

$$
Q\left(t, x^{0}, \vec{x}\right)=\phi\left(t+x^{0}, \vec{x}\right)
$$

The Lagrangian density in $d+1$ dimensions for $Q\left(t, x^{\mu}\right)$ [see Eq. (8)] is

$$
\begin{aligned}
\mathcal{L}\left(t, x^{\mu}\right)= & -\frac{1}{2} \partial_{\mu} Q(t, x) \partial^{\mu} Q(t, x)-\frac{m^{2}}{2} Q(t, x)^{2} \\
& -\frac{g}{3 !} Q(t, x) * Q(t, x) * Q(t, x),
\end{aligned}
$$

where now the derivatives in $*$ are with respect to $x^{\mu}$ $=\left(x^{0}, \vec{x}\right)$. Note that this Lagrangian density is local in the evolution time $t$.

The momentum constraint (10) is given by

$$
\begin{aligned}
\varphi\left(t, x^{\mu}\right)= & P\left(t, x^{\mu}\right)-\delta\left(x^{0}\right) Q^{\prime}(t, x) \\
& +\frac{g}{2 !} \int d x^{\prime} \chi\left(x^{0},-x^{\prime 0}\right) \int d y_{1} d y_{2} \\
& \times K\left(y_{1}-x^{\prime}, y_{2}-x^{\prime}, x-x^{\prime}\right) Q\left(t, y_{1}\right) Q\left(t, y_{2}\right),
\end{aligned}
$$

where $Q^{\prime}(t, x)$ denotes $\partial_{x^{0}} Q\left(t, x^{\mu}\right)$. Here $K$ is the symmetric kernel of three star products:

$$
\begin{aligned}
f(x) * g(x) * h(x)= & \int d y_{1} d y_{2} d y_{3} \\
& \times K\left(y_{1}-x, y_{2}-x, y_{3}-x\right) \\
& \times f\left(y_{1}\right) g\left(y_{2}\right) h\left(y_{3}\right) .
\end{aligned}
$$

The Hamiltonian (6) is

$$
\begin{aligned}
H(t)= & \int d x\left[P(t, x) Q^{\prime}(t, x)-\mathcal{L}(t, x) \delta\left(x^{0}\right)\right] \\
= & \int d x\left[P(t, x) Q^{\prime}(t, x)+\delta\left(x^{0}\right)\left\{-\frac{1}{2} Q^{\prime}(t, x)^{2}\right.\right. \\
& +\frac{1}{2}[\nabla Q(t, x)]^{2}+\frac{m^{2}}{2} Q(t, x)^{2} \\
& \left.\left.+\frac{g}{3 !} Q(t, x) * Q(t, x)^{*} Q(t, x)\right\}\right]
\end{aligned}
$$

The Hamilton equations are

$$
\begin{aligned}
\dot{Q}(t, x)= & Q^{\prime}(t, x) \\
\dot{P}(t, x)= & P^{\prime}(t, x)-\delta^{\prime}\left(x^{0}\right)\left[Q^{\prime}(t, x)\right]_{x^{0}=0}+\delta\left(x^{0}\right) \\
& \times\left\{\nabla^{2} Q(t, x)-m^{2} Q(t, x)\right\} \\
& -\frac{g}{2 !} \int d x^{\prime} d y_{1} d y_{2} \delta\left(x^{\prime 0}\right) \\
& \times K\left(y_{1}-x^{\prime}, y_{2}-x^{\prime}, x-x^{\prime}\right) \\
& \times Q\left(t, y_{1}\right) Q\left(t, y_{2}\right) .
\end{aligned}
$$

The stability of the constraint implies the new constraints (13):

$$
\begin{aligned}
\psi(t, x) \equiv & \left(\nabla^{2}-\partial_{x^{0}}^{2}-m^{2}\right) Q(t, x) \\
& -\frac{g}{2 !} Q(t, x)^{*} Q(t, x)=0, \quad \text { at } \quad x^{0}=0 .
\end{aligned}
$$

By requiring further consistency we have an infinite number of constraints which can be written as, Eq. (14),

$$
\psi(t, x)=0 \text { for }-\infty<x^{0}<\infty .
$$

Using the Hamilton equation (51) for $Q$, Eq. (54) becomes the EL equation 


$$
\left(\nabla^{2}-\partial_{t}^{2}-m^{2}\right) Q(t, x)-\frac{g}{2 !} Q(t, x) * Q(t, x)=0
$$

where $\partial_{x^{0}}$ on $Q$ is replaced by $\partial_{t}$ both in the first term and in the $*$ product. It is the original non-local EL equation (45).

If we write the symplectic form and the Hamiltonian in terms of $Q(t, x)$, Eqs. (34) and (32), we have

$$
\begin{aligned}
\Omega= & \int d x \delta\left(x^{0}\right) \delta Q^{\prime}(t, x) \wedge \delta Q(t, x) \\
& -\frac{g}{4} \int d x \delta\left(Q(t, x)^{*} Q(t, x)\right) \epsilon\left(x^{0}\right) \wedge \delta Q(t, x)
\end{aligned}
$$

and

$$
\begin{aligned}
H= & \int d x \frac{\delta\left(x^{0}\right)}{2}\left\{Q^{\prime}(t, x)^{2}+[\nabla Q(t, x)]^{2}+m^{2} Q(t, x)^{2}\right\} \\
& -\frac{g}{4} \int d x\left[Q(t, x)^{*} Q(t, x)\right] \epsilon\left(x^{0}\right) Q^{\prime}(t, x)
\end{aligned}
$$

These expressions can be rewritten in terms of $\phi(x)$ using Eq. (51), i.e., Eq. (46). In particular the interaction Hamiltonian becomes

$$
H_{i}=-\frac{g}{4} \int d x[\phi(x) * \phi(x)] \epsilon\left(x^{0}\right) \dot{\phi}(x) .
$$

Note that the occurrence of time derivatives of any order in the interaction Hamiltonian is not forbidden in non-local theories. This property is clearly not satisfied by local theories.
Now we can perform the path integral quantization using Eq. (42) to obtain

$$
\begin{aligned}
& \int[d \phi(x)] \exp \left[\int d x \left(\frac{1}{2} \partial_{\mu} \phi(x) \partial^{\mu} \phi(x)-\frac{m^{2}}{2} \phi(x)^{2}\right.\right. \\
& \left.\left.-\frac{g}{3 !} \phi(x) * \phi(x) * \phi(x)\right)\right],
\end{aligned}
$$

from which we read the Lagrangian Feynman rules [23]. They coincide with the ones used in [3]. Therefore, it follows from [3] and [4] that non-commutative $\phi^{3}$ theory with timelike non-commutativity is not unitary while noncommutative $\phi^{3}$ theory with light-like non-commutativity is unitary.

Note added. Recently, Ref. [24] has also considered the Hamiltonian formalism for non-local theories.

\section{ACKNOWLEDGMENTS}

We acknowledge discussions with Luis Alvarez-Gaumé, José Barbón, Jaume Gomis, Esperanza Lopez, and Karl Landsteiner and Luis Alvarez-Gaumé for a careful reading of the manuscript. The work of J.G. is partially supported by AEN98-0431, GC 1998SGR (CIRIT), the work of J.L. is supported by DIGICyT, contract PPB96-0384 and by IEC(SCF), and K.K. is partially supported by a Grant-in-Aid for Scientific Research, No. 12640258 (Ministry of Education Japan).
[1] N. Seiberg, L. Susskind, and N. Toumbas, J. High Energy Phys. 06, 044 (2000).

[2] L. Alvarez-Gaumé and J. L. F. Barbón, hep-th/0006209.

[3] J. Gomis and T. Mehen, Nucl. Phys. B591, 265 (2000).

[4] O. Aharony, J. Gomis, and T. Mehen, J. High Energy Phys. 09, 023 (2000).

[5] N. Seiberg and E. Witten, J. High Energy Phys. 09, 032 (1999).

[6] N. Seiberg, L. Susskind, and N. Toumbas, J. High Energy Phys. 06, 021 (2000).

[7] R. Gopakumar, J. Maldacena, S. Minwalla, and A. Strominger, J. High Energy Phys. 06, 036 (2000).

[8] R. Gopakumar, S. Minwalla, N. Seiberg, and A. Strominger, J. High Energy Phys. 08, 008 (2000).

[9] J. L. F. Barbon and E. Rabinovici, Phys. Lett. B 486, 202 (2000).

[10] I. R. Klebanov and J. Maldacena, hep-th/0006085.

[11] From now on when we will refer to non-local theories as the ones with an infinite number of time derivatives

[12] Non-local theories have arisen in a wide variety of physical problems. A very short list should include: A. D. Fokker, Z. Phys. 58, 386 (1929); A. Pais and G. E. Uhlenbeck, Phys. Rev.
79, 145 (1950); P. Kristensen and C. Moller, K. Dan. Vidensk. Selsk. Mat. Fys. Medd. 27, 7 (1952).

[13] For a discussion about general aspects of non-local theories see D. A. Eliezer and R. P. Wooddard, Nucl. Phys. B325, 389 (1989).

[14] J. Gomis, K. Kamimura, and T. Mateo hep-th/0009158.

[15] J. Llosa and J. Vives, J. Math. Phys. 35, 2856 (1994).

[16] M. Ostrogradski, Mem. Ac. St. Petersbourg 6, 385 (1850).

[17] M. M. Sheikh-Jabbari and A. Shirzad, hep-th/9907055.

[18] C. Batlle, J. Gomis, J. M. Pons, and N. Roman-Roy, J. Math. Phys. 27, 2953 (1986).

[19] The connection with Ostrogradski formalism has also been analyzed in [15] in the context of symplectic mechanics.

[20] R. Marnelius, Phys. Rev. D 10, 2535 (1974).

[21] L. D. Faddeev, Theor. Math. Phys. 1, 1 (1969).

[22] P. Senjanovic, Ann. Phys. (N.Y.) 100, 227 (1976); 209, 248 (1991).

[23] In general the Feynman rules derived from the path integral Lagrangian formalism do not always give the correct Feynman rules; see for example T. D. Lee, Particle Physics and Introduction to Field Theory (Academic Press, New York, 1981).

[24] R. P. Woodard, Phys. Rev. A 62, 052105 (2000). 